\title{
Correction to: Genetic structure and diversity in relation to the recently reduced population size of the rare conifer, Pseudotsuga japonica, endemic to Japan
}

\author{
Satoshi Tamaki ${ }^{1}$ (D) Keiya Isoda ${ }^{2} \cdot$ Makoto Takahashi $^{2} \cdot$ Hiroo Yamada $^{3} \cdot$ Yumiko Yamashita $^{4}$
}

Published online: 28 August 2018

(c) Springer Nature B.V. 2018

\section{Correction to: Conservation Genetics \\ https://doi.org/10.1007/s10592-018-1092-5}

In the original publication, Table 3 was published with incorrect $\mathrm{F}_{\mathrm{IS}}$ values for three populations Kawamatakannnonn, Ootousan and Senbonyama. The updated Table 3 with correct $\mathrm{F}_{\mathrm{IS}}$ values are provided in this Correction.

The original article can be found online at https://doi.org/10.1007/ s10592-018-1092-5.

Satoshi Tamaki

tamakis@affrc.go.jp

1 Tohoku Regional Breeding Office, Forest Tree Breeding Center, Forestry and Forest Products Research Institute, 95

Oosaki, Takizawa, Iwate 020-0621, Japan

2 Genetic Resources Department, Forest Tree Breeding Center, Forestry and Forest Products Research Institute, 3809-1 Ishi, Juo, Hitachi, Ibaraki 319-1301, Japan

3 Kansai Regional Breeding Office, Forest Tree Breeding Center, Forestry and Forest Products Research Institute, 1043, Uetsukinaka, Shoo, Katsuta, Okayama 709-4335, Japan

4 Wakayama Prefectural Forestry Experiment Station, 1504-1 Ikuma, Kamitonda, Wakayama 649-2103, Japan 
Table 3 Measurements of genetic diversity based on six SSR loci in seven populations of Pseudotsuga japonica

\begin{tabular}{lllrlllllll}
\hline Region & Population & Code & $\mathrm{N}$ & \multicolumn{1}{c}{$A$} & $A_{P}$ & $R A^{\mathrm{a}}$ & $A_{R}$ & $H_{O}$ & $H_{E}$ & \multicolumn{1}{c}{$F_{I S}$} \\
\hline Kii Peninsula & Sannokou & K-SAN & 66 & 7.333 & 7 & 18 & 6.263 & 0.657 & 0.647 & -0.011 \\
& Oomata & K-OMA & 90 & 5.333 & 2 & 12 & 4.669 & 0.547 & 0.538 & -0.017 \\
& Kawamatakannon & K-KAW & 101 & 6.833 & 6 & 15 & 5.785 & 0.564 & 0.566 & 0.004 \\
& Ootousan & K-OTO & 55 & 6.667 & 4 & 14 & 5.939 & 0.647 & 0.655 & 0.012 \\
\multirow{5}{*}{ Shikoku Island } & Senbonnyama & S-SEN & 30 & 5.167 & 3 & 10 & 5.167 & 0.467 & 0.472 & 0.011 \\
& Yasudagawayama & S-YAS & 59 & 5.333 & 3 & 10 & 4.791 & 0.558 & 0.548 & -0.020 \\
& Nishinokou & S-NIS & 50 & 5.333 & 4 & 9 & 5.110 & 0.582 & 0.552 & -0.054 \\
\hline
\end{tabular}

$N$ number of samples, $A$ average number of alleles per locus, $A_{P}$ number of private alleles, $R A$ number of rare alleles, $A_{R}$ allelic richness, $H_{O}$ average observed heterozygosity, $H_{E}$ average expected heterozygosity, $F_{I S}$ fixation indices

${ }^{\mathrm{a}}$ Rare alleles correspond to a frequency $<0.05$ 